\title{
Dalfampridine to Improve Balance in Multiple Sclerosis: Substudy from a Randomized Placebo-Controlled Trial
}

\author{
Luca Prosperini ${ }^{1}$ (D) - Letizia Castelli ${ }^{2} \cdot$ Laura De Giglio $^{3,4} \cdot$ Valeria Bonanno $^{4,5} \cdot$ Claudio Gasperini $^{1} \cdot$ Carlo Pozzilli $^{4,5}$
}

Published online: 9 December 2019

(C) The American Society for Experimental NeuroTherapeutics, Inc. 2019

\begin{abstract}
This was a substudy of a randomized, double-blind, placebo-controlled trial originally designed to explore the effect of dalfampridine on information processing speed (2013-002558-64 EU Clinical Trials Register) in patients with multiple sclerosis (MS). A total of 120 patients were originally randomized in a 2:1 ratio to receive dalfampridine $10 \mathrm{mg}$ or placebo twice daily for 12 weeks. Here, we sought to explore the effect of dalfampridine on static balance in single-task and dual-task conditions in a subgroup of 41 patients. They underwent static posturography in quiet standing (single-task) and while performing the Stroop test (dual-task) at randomization (baseline), after 12 weeks and after a 4-week wash-out period. Baseline characteristics of active group $(n=27)$ did not differ from those of placebo group $(n=14)$. Dalfampridine treatment was associated with better balance control than placebo in both single-task $(F=4.80, p=0.034)$ and dual-task $(F=6.42, p=0.015)$ conditions, with small-tomoderate effect sizes (Cohen's $f^{2}=0.122-0.162$ ). The beneficial effect of dalfampridine was not retained 4 weeks after its discontinuation. The rate of accidental falls per month did not differ between the two groups $(p=0.12)$. Our preliminary findings suggest that dalfampridine can be considered a potential option to treat balance impairment due to MS. Larger sample sizes are needed to verify if the beneficial effect of dalfampridine on balance can be translated into a reduced risk of accidental falls.
\end{abstract}

Keywords Multiple sclerosis $\cdot$ dalfampridine $\cdot$ clinical trial $\cdot$ balance $\cdot$ accidental falls

\section{Introduction}

In a double-blind, randomized, placebo-controlled trial (2013002558-64 EU Clinical Trials Register), we recently provided class I evidence that dalfampridine improves information processing speed in patients with multiple sclerosis (MS) who

Luca Prosperini

luca.prosperini@gmail.com

1 Dept. of Neurosciences, S. Camillo-Forlanini Hospital, C.ne Gianicolense 87, 00152 Rome, Italy

2 Istituto di Ricerca e Cura a Carattere Scientifico (IRCCS) Fondazione Don Carlo Gnocchi, -, Piazzale Morandi 6, 20121 Milano, Italy

3 Neurology Unit, S. Filippo Neri Hospital, Via G. Martinotti 20, 00135 Rome, Italy

4 S. Andrea Hospital, Sapienza University, Via di Grottarossa 1035, 00189 Rome, Italy

5 Dept. of Human Neuroscience, Sapienza University, Viale dell'Università 30, 00185 Rome, Italy present deficit in sustained attention, as detected with the Symbol Digit Modalities Test (SDMT) [1]. Impairment in sustained attention was defined as a score in the SDMT below the 10th percentile of normative values of the Italian Population [2].

Attention and executive functions play a crucial role in balance control and gait, especially when a motor task is performed simultaneously to a cognitive task that can unmask the so-called cognitive-motor interference phenomenon $[3,4]$. This raises the hypothesis of an overlap between network subserving some cognitive functions and postural control [4-6]. Impaired attention and verbal fluency have been associated with increased risk of accidental falls in patients with MS [7], likely due to damage to areas of the central nervous system that not only are crucial for gait and postural control $[6,8]$ but also are involved in cognitive processes [9].

Unfortunately, no pharmacological treatment is available today for balance impairment due to MS. On the basis of its beneficial effect on information processing speed, our work hypothesis is that dalfampridine can potentially have an effect even on balance, as previously 
suggested [10-13]. Here, we report the findings of a substudy aimed at exploring the effect of dalfampridine versus placebo on balance in a subgroup of patients who participated in the original trial [1].

\section{Methods}

\section{Study Design}

This was a substudy of a randomized, double-blind, placebocontrolled trial in which 120 patients with MS were randomized in a 2:1 ratio to receive dalfampridine $10 \mathrm{mg}$ or placebo twice daily for 12 consecutive weeks [1]. The trial was conducted in accordance with the Declaration of Helsinki and its subsequent amendments, Good Clinical Practice, and applicable regulatory requirements. The protocol was approved by the Ethical Committee of Sapienza University in Rome and registered on EU Clinical Trials Register with the number 2013-002558-64. All patients signed an informed consent prior to any study-related procedure.

Eligibility criteria included the following: (i) diagnosis of MS according to the revised McDonald criteria [14], regardless of disease course (relapsing-remitting, primary progressive, or secondary progressive) [15]; (ii) age ranging from 18 to 65 years (inclusive); (iii) no clinical relapse in the previous 60 days; (iv) no moderate or severe depression according to Beck Depression Inventory-II (BDI-II), corresponding to a cutoff score of 19 [16]; (v) no history of psychiatric illnesses; (vi) no history of seizures; (vii) no therapy change or introduction of new drugs in the previous month, including medication for mood, fatigue, or cognition. Further details on the inclusion/exclusion criteria and study design are published elsewhere [1].

As additional criteria to be included in this substudy, patients were also required to be able to stand upright for at least $180 \mathrm{~s}$ without any support and to be still ambulant, i.e., scoring less than 6.0 at the Expanded Disability Status Scale (EDSS) [17].

Patients from the original trial who agreed to participate in this substudy were evaluated with static posturography in quiet standing (single-task) and while performing the Stroop test (dual-task) according to an established experimental design [5] by using a laboratory-grade force platform (ProKin PK254P, Tecnobody, Bergamo, Italy; http://www.tecnobody.it). Patients were instructed to maintain their balance for $30 \mathrm{~s}$ as steady as possible under eyes opened (single-task condition) and while performing the Stroop test (dual-task condition) presented on a printout located $2 \mathrm{~m}$ from the force platform. They were evaluated at randomization (baseline or T0), at the end of the 12-week period of treatment with dalfampridine or placebo (T1), and after 4 weeks of wash-out period (T2).
We established the protocol for collecting posturographic data when the enrolment for the original trial had already been started; therefore, participation in this substudy was not offered to all patients recruited in the original trial.

\section{Endpoints}

The main endpoint was the postural sway in single-task and dual-task conditions detected at static posturography, i.e., the sum of displacements (millimeters) of the body's center of pressure on a standard force platform measured in $30 \mathrm{~s}$; larger sway indicates worse standing balance [5]. Static posturography provides computer-based and objective instrumented measurements of balance that are highly reliable [18-20] and valid, not only in differentiating healthy controls from patients with MS [21] but also in stratifying patients at different levels of disabilities [22, 23]. Furthermore, static standing balance measurements are more sensitive and accurate than common clinical scales in predicting future accidental falls [18]. On the other hand, data on minimal detectable change and minimal importance difference for posturographic measures are still lacking.

As additional endpoint, we considered the number of accidental falls collected by patient diary.

\section{Statistical Analysis}

Given the exploratory nature of this study, neither sample size estimation nor correction for multiplicity was done. Between-group baseline differences were tested by the Mann-Whitney $U$ test or the Fisher test for continuous and categorical variables, respectively. Semiparametric repeated measures analyses of variance (RM-ANOVAs) were carried out to explore the interaction effect of time by group (dalfampridine $v s$. placebo) on postural sway in single-task and dual-task conditions (dependent variables). Semiparametric RM-ANOVAs were based on ranks to test differences in the distribution of data, thus providing more robust results [24]. Given that Mauchly's test indicated that the sphericity assumption of RM-ANOVAs had been violated $\left(\chi^{2}>20.1, p<0.001\right)$, we applied the lower-bound correction. Effect size (ES), expressed as Cohen's $f^{2}$, was also reported. Between-group difference in the incidence rate ratio (IRR) of accidental falls per month was investigated by a Poisson regression model. Correlations between change in postural sway and number of accidental falls were tested by the Spearman rank coefficients. Lastly, we explored whether the response to dalfampridine according to SDMT (defined as an increase $>4$ points in the raw score [25]) correlated with improvement in postural sway. $p$ values less than 0.05 in either direction were considered as statistically significant. 


\section{Results}

A total of 120 patients were randomized in the original trial; of them, 41 (27 women and 14 men) agreed to participate in the additional balance evaluations (see the study flowchart in Fig. 1). They had a mean \pm standard deviation (SD) age of $51 \pm 7.7$ years, disease duration of 17.0 \pm 9.6 years, and median EDSS score of 4.0 (interval, 1.5 to 5.5 ). There was no significant difference between the 41 patients enrolled in this substudy and those who did not $(n=79)$ in terms of sex, age, formal education, and disease duration, whereas excluded patients had a worse EDSS score $(p<0.05)$. No patients with primary progressive disease course participated in this substudy.

Demographic and clinical characteristics at study enrolment were well balanced across groups (Table 1). The summary of the main study findings is shown in Table 2 . We found a significant effect of time by group interaction in both single-task condition $(F=4.80, p=0.034 ; \mathrm{ES}=0.122)$ and dual-task condition $(F=$ $6.42, p=0.015 ; \mathrm{ES}=0.162$ ) on postural sway; this indicates a balance improvement in the active group but not in the placebo group at $\mathrm{T} 1$, whereas the between-group difference was no longer significant at T2 (Fig. 2). Postural sway in the singletask condition did not merely return to baseline values, but worsened slightly after the wash-out period in the active group, whereas there was a nonsignificant trend towards balance worsening under both single-task and dual-task conditions in the placebo group over the entire study period (see also Fig. 2). These latter findings were mainly attributable to balance deterioration in patients with secondary progressive disease course.

The rate of accidental falls per month was higher in the placebo group than that in the active group (0.33 vs. 0.22), but there was no significant between-group difference (IRR = $1.5, p=0.12$ ). However, we found that the more the improvement (reduction) in postural sway under dual-task condition at $\mathrm{T} 1$, the fewer the number of accidental falls over the entire study period in the active group ( $\rho=-0.41, p=0.035)$. Accordingly, there was a direct correlation between the worsening (increase) in postural sway under the dual-task condition and the number of accidental falls occurred over the entire study period in the placebo group ( $\rho=0.62, p=0.018$ ). Changes in postural sway under the single-task condition did not correlate with accidental falls in both groups.

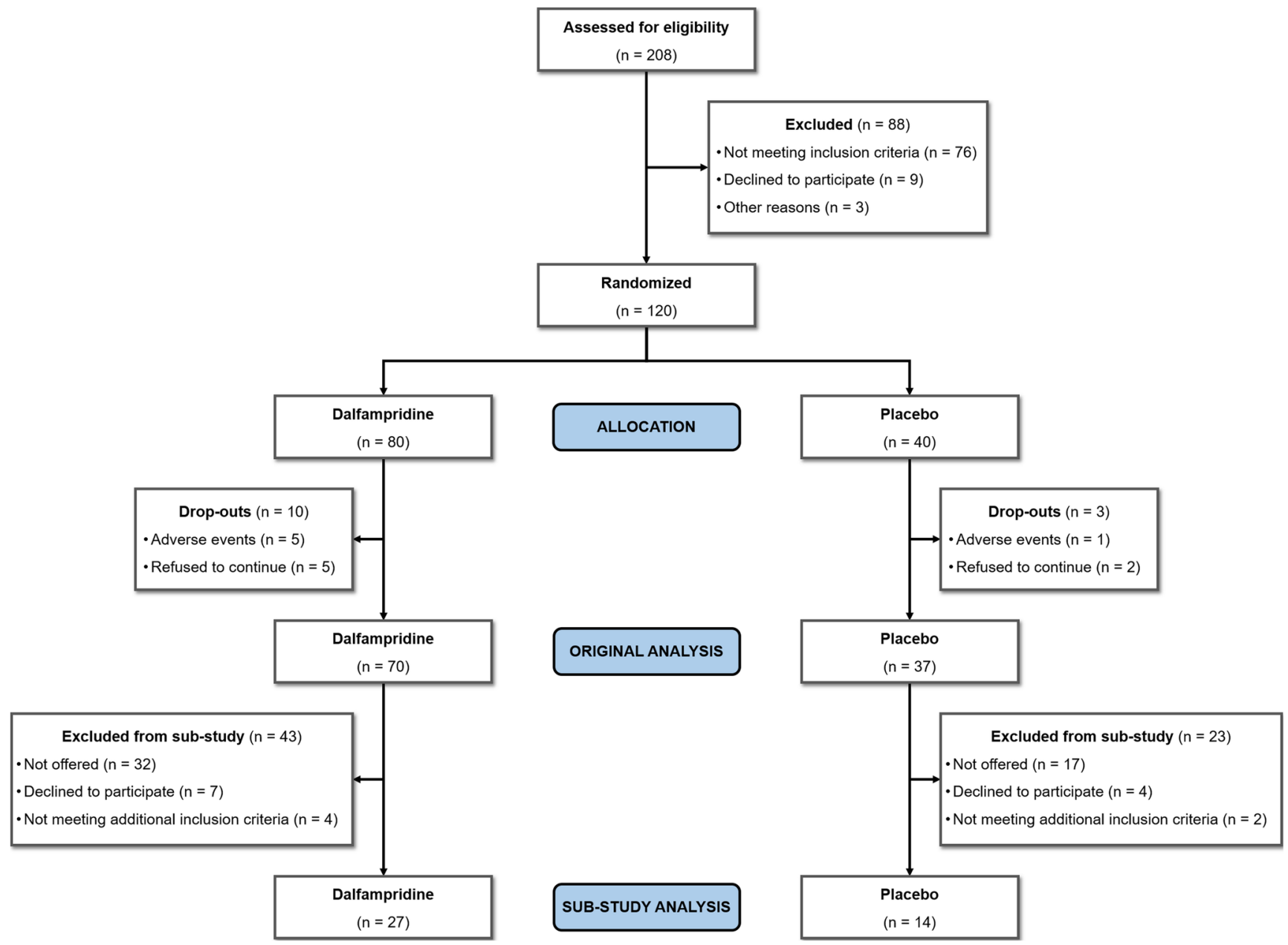

Fig. 1 Study flowchart of patients' disposition 
Table 1 Characteristics of study sample at randomization $(n=41)$

\begin{tabular}{lll}
\hline & Dalfampridine & Placebo \\
\hline$N$ & 27 & 14 \\
Female:male, $n$ & $18: 9$ & $9: 5$ \\
Age, years & $51.3(7.1)$ & $50.3(8.9)$ \\
Body mass index, $\mathrm{kg} / \mathrm{m}^{2}$ & $22.6(3.2)$ & $24.6(4.7)$ \\
Formal education, years & $12.7(4.0)$ & $14.4(3.1)$ \\
Disease duration, years & $18.7(9.6)$ & $14.4(9.4)$ \\
Median (interval) EDSS score & $4.0(1.5$ to 5.5) & $4.0(2.0$ to 5.5) \\
Relapsing-remitting:secondary progressive disease course, $n$ & $24: 3$ & $12: 2$ \\
Postural sway (single-task), mm & $361(196)$ & $348(198)$ \\
Postural sway (dual-task), mm & $475(252)$ & $444(243)$ \\
Median (interval) self-reported accidental falls in the past three months & $0(0$ to 10) & 0.5 (0 to 7) \\
\hline
\end{tabular}

$E D S S=$ Expanded Disability Status Scale. Values are mean ( \pm standard deviation), unless indicated otherwise; all $p$ values $>0.2$
Overall, we classified 26 out of 41 patients $(63.4 \%)$ as responders to dalfampridine according to SDMT: 20 in the active group and 6 in the placebo group $(p=0.049)$. Postural sway improved in dalfampridine responders than in nonresponders under both single-task condition (mean $\pm \mathrm{SD}$ changes, $-15.6 \pm 26.2$ versus $-0.6 \pm 38.3 \mathrm{~mm} ; p=0.056$ ) and dual-task condition (mean \pm SD changes, $-22.1 \pm 45.0$ versus $14.1 \pm 56.8 \mathrm{~mm} ; p=0.062)$.

\section{Discussion}

This study suggests that, among patients with MS and impaired sustained attention, a 12 -week treatment with dalfampridine is associated with more improved standing balance than placebo under both single-task and dual-task conditions (class II evidence). However, the effect of dalfampridine on standing balance was only small to moderate and did not translate directly into a reduced incidence of accidental falls. This latter finding confirms the multifactorial causes underlying accidental falls in MS, which goes beyond the mere deficit in postural control [26]. On the other hand, we also found an indirect relationship between improved postural control and accidental falls that encourages further investigation. In this regard, the present study allows a correct estimation for calculating the sample size of a hypothetical trial aimed at exploring the effect of dalfampridine on accidental falls. Interestingly, we found that patients classified as responders according to SDMT [25] improved also their postural sway, thus further corroborating the hypothesis of an overlap between brain network subserving sustained attention and postural control [3-6, 9]. Another possible explanation that connects the concomitant improvement in balance and information processing speed is the possibility of enhanced visual acuity and ocular motor functions promoted by dalfampridine $[27,28]$. In fact, if on one hand patients with MS are strictly dependent on visual cues while maintaining their balance [21, 29], on the other hand, visual scanning is essential for SDMT execution [25].

As observed for information processing speed [1], the improvement in postural control was not retained after treatment discontinuation. This is expected given the prompt and reversible proposed mechanism of action of dalfampridine involving an enhanced conduction in demyelinated pathways via blockade
Table 2 Summary of study findings $(n=41)$

\begin{tabular}{lllll}
\hline & & T0 & T1 & T2 \\
\hline Single-task condition & & & & \\
Absolute value, mean (SD) & Dalfampridine $(n=27)$ & $361(196)$ & $325(174)$ & $386(199)$ \\
& Placebo $(n=14)$ & $348(198)$ & $362(201)$ & $381(201)$ \\
Mean change from baseline, $\%$ & Dalfampridine $(n=27)$ & N/A & -9.8 & +6.9 \\
& Placebo $(n=14)$ & & +4.0 & +9.5 \\
Dual-task condition & & & & \\
Absolute value, mean (SD) & Dalfampridine $(n=27)$ & $475(252)$ & $430(239)$ & $481(266)$ \\
& Placebo $(n=14)$ & $444(243)$ & $475(277)$ & $488(241)$ \\
Mean change from baseline, $\%$ & Dalfampridine $(n=27)$ & N/A & -9.5 & +1.3 \\
& Placebo $(n=14)$ & & +7.0 & +9.9 \\
\hline
\end{tabular}

$S D=$ standard deviation 
Fig. 2 Postural sway variations over the study period (primary endpoint), expressed as mean and $95 \%$ confidence intervals. $* p<$ 0.05 versus $\mathrm{T} 0$

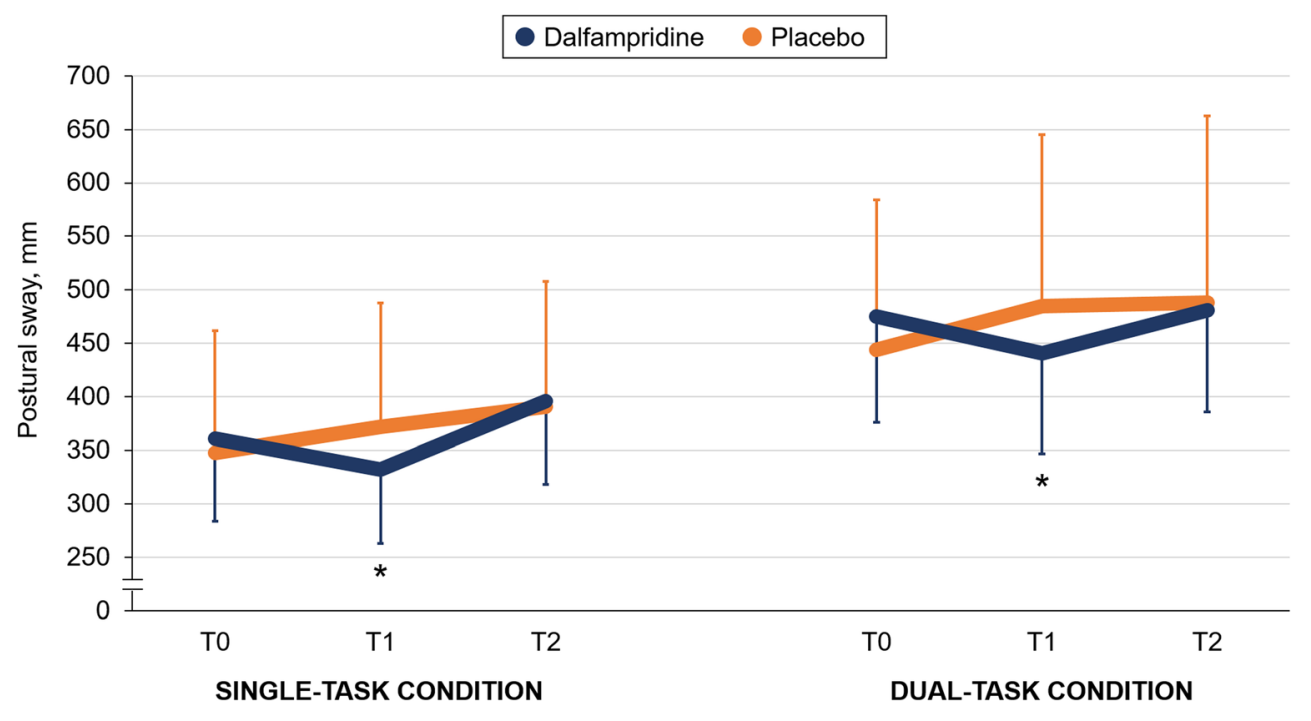

of voltage-dependent potassium channels. Regarding the improvement in balance control, whose efficiency is strictly dependent on cerebellum and its connections, we acknowledge that dalfampridine is also capable to modulate the Purkinje cell excitability and to normalize the irregular firing rate, so that the inhibitory influence of the cerebellar cortex on vestibular and deep cerebellar nuclei is restored [30-33].

We observed a trend towards balance deterioration in the placebo group over the entire study period, in spite of its short duration (16 weeks). In this regard, we should take into account that computer-based measures of balance and gait are more sensitive to subtle clinical progression related to neurodegenerative processes than EDSS and other clinical scales $[34,35]$. In the active group, measures of postural sway (especially under single-task condition) did not return to baseline level, but deteriorated slightly during the wash-out period after dalfampridine interruption. This latter phenomenon has been already described in a previous study in which discontinuation of treatment resulted in worsening beyond pretreatment level of self-reported walking ability [11]. The inclusion of patients with progressive disease course would explain these findings in the aforementioned MOBILE trial [11] and even in our study.

Data from the present study should be interpreted cautiously, as the original trial was not designed to explore the effect of dalfampridine on balance. Participants were originally selected by the presence of deficit in sustained attention [1] and static posturography assessment was reserved only to patients able to stand upright for at least $180 \mathrm{~s}$ [5], thus providing data on subjects with a relatively advanced disability. Therefore, while being encouraging, our findings should be considered only preliminary and cannot be generalized to all patients with MS. Moreover, the sample size was smaller than that of the original trial, thus leading to reduced statistical power.
In conclusion, dalfampridine may represent a potential option to treat balance impairment due to MS. However, given the number of potential methodological weaknesses and the lack of a direct effect on accidental falls, our study is not conclusive. Therefore, the role of dalfampridine in the management of MSrelated balance impairment deserves future research based on larger sample size and/or longer term follow-up.

Required Author Forms Disclosure forms provided by the authors are available with the online version of this article.

Funding Information The original study was funded by Biogen (Investigator initiated trial [ITT], grant number CUP B85I1700033000).

\section{Compliance with Ethical Standards}

Ethics Statement The trial was conducted in accordance with the Declaration of Helsinki and its subsequent amendments, Good Clinical Practice, and applicable regulatory requirements. The protocol was approved by the Ethical Committee of Sapienza University in Rome and registered on EU Clinical Trials Register with the number 2013-002558-64. All patients signed an informed consent prior to any study-related procedure.

Conflict of Interest LP: consulting and lecture fees and travel grants from Almirall, Biogen, Novartis, Genzyme, Roche, and Teva; and research funding from Associazione Italiana Sclerosi Multipla and Genzyme.

LC: consulting fees from Almirall.

LDG: travel grants from Biogen, Genzyme, Teva, and Novartis; consulting fees from Genzyme, Merck, Novartis, and Biogen.

VB: nothing to disclose.

CG: consulting fees from Bayer and Biogen, and lecture fees from Biogen, Bayer, Genzyme, Merck, Novartis, and Teva.

$\mathrm{CP}$ : consulting and lecture fees and research funding and travel grants from Almirall, Actelion, Bayer, Biogen, Genzyme, Merck, Novartis, Roche, and Teva.

Disclaimer The funder provided financial resources for the study conduction and the dalfampridine/placebo tablets, but had no role in study design, data collection, data analysis, data interpretation, or writing of the report. 


\section{References}

1. De Giglio L, De Luca F, Gurreri F, et al. Effect of dalfampridine on information processing speed impairment in multiple sclerosis. Neurology. 2019.

2. Amato MP, Portaccio E, Goretti B, et al. The Rao's Brief Repeatable Battery and Stroop Test: normative values with age, education and gender corrections in an Italian population. Mult Scler 2006; 12: 787-793.

3. Leone C, Patti F, Feys P. Measuring the cost of cognitive-motor dual tasking during walking in multiple sclerosis. Mult. Scler. 2015; 21(2):123-131.

4. Leone C, Feys P, Moumdjian L, et al. Cognitive-motor dual-task interference: a systematic review of neural correlates. Neurosci. Biobehav. Rev. 2017; 75:348-360.

5. Prosperini L, Castelli L, De Luca F, et al. Task-dependent deterioration of balance underpinning cognitive-postural interference in MS. Neurology. 2016; 87(11):1085-1092.

6. Kalron A, Allali G, Achiron A. Cerebellum and cognition in multiple sclerosis: the fall status matters. J Neurol. 2018; 265: 809-816

7. Kalron A. The relationship between specific cognitive domains, fear of falling, and falls in people with multiple sclerosis. Biomed Res Int. 2014; 2014: 281760.

8. Prosperini L, Sbardella E, Raz E, et al. Multiple sclerosis: white and gray matter damage associated with balance deficit detected at static posturography. Radiology. 2013; 268(1):181-189.

9. Ruggieri S, Fanelli F, Castelli L, et al. Lesion symptom map of cognitive-postural interference in multiple sclerosis. Mult Scler 2018; 24: 653-662.

10. Fjeldstad C, Suárez G, Klingler M, et al. Dalfampridine effects beyond walking speed in multiple sclerosis. Int. J. MS Care. 2015; 17(6):275-283.

11. Hupperts R, Lycke J, Short C, et al. Prolonged-release fampridine and walking and balance in MS: randomised controlled MOBILE trial. Mult. Scler. 2016; 22(2):212-221.

12. Filli L, Zorner B Kapitza S, et al. Monitoring long-term efficacy of fampridine in gait impaired patients with multiple sclerosis. Neurology. 2017; 88:832-841.

13. Albrecht P, Bjorna IK, Brassat D, et al. Prolonged-release fampridine in multiple sclerosis: clinical data and real-world experience. Report of an expert meeting. Ther Adv Neurol Disord. 2018; 11: doi:https://doi.org/10.1177/1756286418803248.

14. Thompson AJ, Banwell BL, Barkhof F, et al. Diagnosis of multiple sclerosis: 2017 revisions of the McDonald criteria. Lancet Neurol 2018; 17: 162-173.

15. Lublin FD, Reingold SC, Cohen JA, et al. Defining the clinical course of multiple sclerosis: the 2013 revisions. Neurology. 2014; 83: 278-286.

16. Beck AT, Steer RA, Ball R, Ranieri W. Comparison of Beck depression inventories -IA and -II in psychiatric outpatients. J Pers Assess 1996; 67: 588-597.

17. Kurtzke JF. Rating neurologic impairment in multiple sclerosis: an Expanded Disability Status Scale (EDSS). Neurology. 1983; 33: 1444-1452.

18. Prosperini L, Fortuna D, Giannì C, Leonardi L, Pozzilli C. The diagnostic accuracy of static posturography in predicting accidental falls in people with multiple sclerosis. Neurorehabil Neural Repair. 2013; 27: 45-52.
19. Wajda DA, Motl RW, Sosnoff JJ. Three-month test-retest reliability of center of pressure motion during standing balance in individuals with multiple sclerosis. Int J MS Care. 2016; 18: 59-62.

20. Melillo F, Di Sapio A, Martire S, Malentacchi M, Matta M, Bertolotto A. Computerized posturography is more sensitive than clinical Romberg test in detecting postural control impairment in minimally impaired multiple sclerosis patients. Mult Scler Relat Disord. 2017; 14: 51-55.

21. Cattaneo D, Jonsdottir J. Sensory impairments in quiet standing in subjects with multiple sclerosis. Mult Scler. 2009;15: 59-67.

22. Kalron A, Nitzani D, Achiron A. Static posturography across the EDSS scale in people with multiple sclerosis: a cross sectional study. BMC Neurol. 2016; 16: 70.

23. Kalron A, Givon U, Frid L, Dolev M, Achiron A. Static posturography and falls according to pyramidal, sensory and cerebellar functional systems in people with multiple sclerosis. PLoS One. 2016; 11: e0164467.

24. Zhuang Y, Guan Y, Qiu L, et al. A novel rank-based non-parametric method for longitudinal ordinal data. Stat. Methods Med. Res. 2018; 27(9):2775-2794.

25. Benedict RHB, DeLuca J, Phillips G, et al. Validity of the Symbol Digit Modalities Test as a cognition performance outcome measure for multiple sclerosis. Mult Scler 2017; 23:721-733.

26. Giannì C, Prosperini L, Jonsdottir J, Cattaneo D. A systematic review of factors associated with accidental falls in people with multiple sclerosis: a meta-analytic approach. Clin Rehabil. 2014; 28: 704-16.

27. Claassen J, Spiegel R, Kalla R et al. A randomised double-blind, cross-over trial of 4-aminopyridine for downbeat nystagmuseffects on slowphase eye velocity, postural stability, locomotion and symptoms. J Neurol Neurosurg Psychiatry. 2013; 84: 1392 1399.

28. Horton L, Conger A, Conger D, et al. Effect of 4-aminopyridine on vision in multiple sclerosis patients with optic neuropathy. Neurology. 2013; 80: 1862-1866.

29. Prosperini L, Leonardi L, De Carli P, Mannocchi ML, Pozzilli C. Visuo-proprioceptive training reduces risk of falls in patients with multiple sclerosis. Mult Scler. 2010; 16: 491-499.

30. Kalla R, Teufel J, Feil K, Muth C, Strupp M. Update on the pharmacotherapy of cerebellar and central vestibular disorders. J. Neurol. 2016; 263 Suppl 1:S24-29.

31. Strupp M, Kalla R, Glasauer S et al. Aminopyridines for the treatment of cerebellar and ocular motor disorders. Progr Brain Res 2008; 171: 535-541.

32. Schniepp R, Jakl V Wuehr M et al. Treatment with 4-aminopyridine improves upper limb tremor of a patient with multiple sclerosis: a video case report. Mult Scler 2012; 19: 506-508.

33. Schmidt S. Fampridine improves severe dysarthria in secondaryprogressive multiple sclerosis. Mult Scler 2013; 19: 511.

34. Galea MP, Cofré Lizama LE, Butzkueven H, Kilpatrick TJ. Gait and balance deterioration over a 12-month period in multiple sclerosis patients with EDSS scores $\leq 3.0$. NeuroRehabilitation. 2017; 40: 277-284.

35. Filli L, Sutter T, Easthope CS, et al. Profiling walking dysfunction in multiple sclerosis: characterisation, classification and progression over time. Sci Rep. 2018; 8: 4984.

Publisher's Note Springer Nature remains neutral with regard to jurisdictional claims in published maps and institutional affiliations. 